\title{
Based on Otsu thresholding Roberts edge detection algorithm research
}

\author{
JinWei Tao \\ College of Electrical and Information Engineering, \\ North University of Nationalities. \\ Yinchuan, China \\ 348688297@qq.com \\ HaiLong Xie \\ College of Electrical and Information Engineering, \\ North University of Nationalities. \\ Yinchuan, China \\ sddtxhlong@163.com
}

\author{
JingZhi Cai \\ College of Electrical and Information Engineering, \\ North University of Nationalities \\ Yinchuan, China \\ cjz410@163.com \\ Xin Ma \\ College of Electrical and Information Engineering, \\ North University of Nationalities. \\ Yinchuan, China \\ kuailebaobei624@126.com
}

\begin{abstract}
In this paper, based on the theory of Roberts edge detection operator, as Roberts algorithm is sensitive to noise and it requires artificial specified threshold and other issues, through the image beforehand Otsu processing division after the formation of binary image, and then after Roberts operator sub-processing through matlab simulation experiments, experimental results obtained . Experimental results show that, after Otsu thresholding ideal image after Roberts operator edge extraction, whether the continuity of the image edge information or anti- noise ability has greatly improved.
\end{abstract}

Keywords- Otsu method; Image processing; edge detection ; edge detection operator

\section{INTRODUCTION}

Digital image edge detection is area of image segmentation, target recognition, area shape extraction is very important, and other areas of the image analysis, the basis of extracting image features and image recognition is an important attribute. In image understanding and analysis, often the first step is the edge detection, it has become one of the most active subject in machine vision research, occupies an important position in the engineering application ${ }^{[1]}$.

The so-called edge is those pixels whose the surrounding blocks with a step change or a change in the roof of the collection of those pixels. Edge of the object is the reflection of Gray discontinuity. The classic image edge extraction method is to examine the gray change of every pixel in a certain field , using edge adjacent to a first or second order directional derivative variation, with a simple method to detect the edge. Such method is called operator method of local edge detection.

Using edge detection for image segmentation, the basic idea is to detect the image edge points, then according to certain strategy connected into a contour edge points, thus constitute the segmented regions.Because the edge is to extract the dividing line between target and background, extract the edge to separate the target and background, as a result, the edge detection technology.

Typical edge detection operators have Sobel operator, Canny operator, Roberts operator, Prewitt operator, Laplacian operator and so on.First, This study was carried out by Otsu thresholding segmentation on the image ,then use the Roberts operator on the image edge detection ,it is an improved algorithm ${ }^{[2]}$.

\section{THE TRADITIONAL ROBERTS EDGE DETECTION ALGORITHM}

\section{A. Roberts Operator is Introduced}

Roberts edge operator is a kind of diagonal deviation calculation method of the gradient, the size of the gradient represents the strength of the edge, the direction of the gradient and marginal, vertical.

Edge detection operator based on any vertically to each pair of the principle of difference can be used to calculate the gradient, the diagonal direction of the difference of the two adjacent pixels, that is:

$$
\left.\begin{array}{c}
\Delta_{x} f=f(i, j)-f(i+1, j+1) \\
\Delta_{y} f=f(i, j+1)-f(i+1, j)
\end{array}\right\}
$$


The convolution operator as follows:

$$
\begin{array}{cc}
{\left[\begin{array}{cc}
1 & 0 \\
0 & -1
\end{array}\right]} & {\left[\begin{array}{cc}
0 & -1 \\
1 & 0
\end{array}\right]} \\
\Delta_{x} f & \Delta_{y} f
\end{array}
$$

And after, it is easy to calculate the Roberts gradient amplitude, appropriate threshold $\mathrm{TH}$, the judge $>\mathrm{TH},(\mathrm{i}, \mathrm{j})$ is a step edge points, for the edge image ${ }^{[3]}$.

\section{B. The Advantages and Disadvantages of Roberts Operator}

Roberts edge operator in the horizontal and vertical direction effect is better, the algorithm is simple, fast calculation speed, but is sensitive to noise, detecting the edge of the coarser discontinuity ${ }^{[4]}$.

Roberts operator defects as follows:

- Only the difference between the adjacent pixels on the diagonal gradient amplitude detection, does not take into account the situation of the horizontal and vertical adjacent pixels adjacent pixels, and is more sensitive to noise.

- Local noise detection operator cannot eliminate, also lost the local edge of grey value changes slowly, which leads to the target object contour edge discontinuity.

- The edge of the measured proved to be more delicate, but not enough ideal for special occasions.

III. ROBERTS EDGE DETECTION BASED ON DAJIN METHOD OF THRESHOLD SEGMENTATION ALGORITHM IS PROPOSED

\section{A. Dajin Method of Threshold Segmentation Principle}

Dajin method is put forward by dajin (OTSU) in $1979^{[5]}$. Is the main principle, give an image $\mathrm{I}, \mathrm{T}$ remember the foreground and background segmentation for the image threshold, the foreground image for percentage points, the average gray level for; Background image for the percentage points, the average grey value is. The total average gray level for the image $u_{t}=w_{0} * u_{0}+w_{1} * u_{1}$ Gray levels from the smallest to the largest grey value traverse $\mathrm{T}$, When $\mathrm{T}$ such that $\sigma^{2}=w_{0} *\left(u_{0}-u_{t}\right)^{2}+w_{1} *\left(u_{1}-u_{t}\right)^{2}$ the maximum variance, $\mathrm{T}$ makes maximum variance, $\mathrm{T}$ is the best threshold segmentation. Variance of gray scale distribution uniformity of one dimension, and the value, the greater the variance shows that the larger the difference of the two parts of the image, as part of the fault current is divided into background or part of the background is divided into outlook will lead to wrong two parts difference is small, therefore, makes the biggest variance ${ }^{[6]}$.

\section{B. Edge Detection Algorithm and Steps}

The algorithm of this paper is, first of all for our image grayscale pretreatment, dajin method was carried out on the processed image threshold segmentation, the segmentation of image edge detection for the image we need. Through such improvement, we will be the original image to image segmentation, through the foreground and background difference better show the characteristics of the edge points, make we are in the edge detection effect is better. Specific steps are as follows:

- $\quad$ The original image gray pretreatment, adding noise.

- We will use Otsu threshold method for the segmentation on preprocessed image, then We got split images.

- On Roberts edge detection after the image segmentation, The image is presented after edge detection.

\section{THE EXPERIMENTAL RESULTS AND ANALYSIS}

In Matlab2010 environment, respectively to the classic figure LENA and actual filming FANG tested in this paper, the edge detection algorithm, and compared with the traditional Roberts operator edge detection of image effects are compared.

Figure 1 is the compare of the traditional algorithm and the improved algorithm renderings, Compared to the traditional Roberts algorithm, we can see that the improved edge extraction algorithm works better at the detection of the details of the person's face, and the character background detail can also be relatively detected by the improved algorithm renderings and edge information detected continuity has been improved.

Figure 2 is a application of the improved algorithm renderings in the actual shooting chart, Compared to the traditional Roberts algorithm, we can see that the facial expressions's details of figure FANG shows better after the work of the improved edge extraction algorithm, the FANG overall continuity of the edge is also improved by the work of traditional operators.

Figure 3 is a picture which added noise to the original grayscale image before edge detection operator, as roberts own characteristics which is sensitive to noise, then we could only get little edge information by the operation of the traditional roberts, while, on the contrary, the edge information extracted by improve algorithm can be more clearly seen in the same situation, and the noise is suppressed to some extent.

Figure 4 is a actual shooting chart which is also added noise before edge detection. As can be seen from Figure 4, the result of edge detection algorithm is better than the one of traditional renderings roberts operator, on the other hand 
,the overall image of FANG can be detected and the resolution is better.

Can be seen from figure 1 and figure 2, Roberts operator,the traditional gray image edge detection, which only get less edge details and low edge continuity,co mpared with the traditional Roberts operator edge detection, this paper improved Roberts operator edge detection ability obtained the very big enhancement, edge continuity has been enhanced, the image can also be good for many details. And you can see as shown in figure 3 , figure 4 , after the original gray image with noise, as a result of the traditional Roberts operator is sensitive to noise, so almost no image edge is detected, and the algorithm of edge detection effect is very obvious, to a certain degree, the improved algorithm is visible.

\section{CONCLUDES}

This paper analyzed the shortcomings of traditional Roberts edge detection operator and tried to put forward the Roberts edge detection algorithm which based on Otsu threshold segmentation and compared Roberts with traditional edge detection operators .Compared each of the four experimental map, you can see this new method of Roberts edge detection operator which compared with traditional edge detection methods proved indeed feasible in edge detection and edge detection with a continuous clear edge, etc., which to a certain extent make up Roberts operator sensitivity to noise with a certain noise immunity.

\section{REFERENCES}

[1] hang Honggang, Chen guang. Image processing and recognition [M]. Beijing: Beijing university of posts and telecommunications publishing house, 2006.

[2] gonzalez. Digital image processing [M]. 2 edition. Beijing: electronic industry press, 2003.

[3] Zhang Kaili. Liu. Edge detection technology development research [J]. Journal of kunming university of science and technology, 2000.

[4] Pan Xiaying. Image edge detection technology research [D]. Xi 'an university of science and technology, 201

[5] ZhangYuJin. Image segmentation [M]. Beijing: science press, 2001.

[6] Yao Min. Digital image processing [M]. Beijing: mechanical industry publishing house, 2005.

[7] Zhou Peide. Computational geometry algorithm analysis and design [M]. Beijing: tsinghua university press, 2000.

[8] Wang Zhi, the game first. An adaptive edge detection method based on the theory of the Canny [J]. Chinese journal of graphics and graphics, 2004

[9] Wang Yue he Wang Qiuguang. Based on the information of the $2 \mathrm{~d}$ graphics edge detection threshold segmentation method [J]. Chinese journal of image and graphics, 2007.

[10] Li Bicheng, Peng Tianjiang Peng Bo, etc. Intelligent image processing technology [M]. Beijing electronic industry Press.

[11] Kang Mu, Xu Qinggong etc. A Roberts adaptive edge detection method. Journal of xi 'an jiaotong university, 2008

[12] Digital image processing and analysis [M]. Beijing: Beijing university of aeronautics and astronautics press, 2007
[13] Gaocheng, etc. The MATLAB image processing and application [M]. 2 edition. Beijing: national defence industry press, 2007.

[14] Zhu Zhu. Edge detection technology research [D]. Suzhou university, 2010

[15] Zhang Chunxue. Image edge detection method research [D]. Southern Yangtze university, 2011

[16] zhang jie. Digital image edge detection technology research [D]. Hefei university of technology, 2009

[17] Ren Wenjie. Image edge detection method research [D]. Shandong university, 2008

[18] Wang Yue he Wang Qiuguang. Based on the graphic twodimensional threshold segmentation method of edge detection information [J]. Chinese journal of image and graphics, 2007.

[19] Yang Huaiyi. Image segmentation algorithm in the application research [J]. Computer simulation, 2012.

[20] Xu Changxin Peng Guohua. The fast algorithm of two-dimensional Otsu thresholding methods [J]. Journal of computer applications, 2012.

[21] Long Jianwu ShenXuan Beijing, Chen Haipeng. Adaptive minimum error threshold segmentation algorithm [J]. Journal of automation, 2012. 
Experiment result figure:

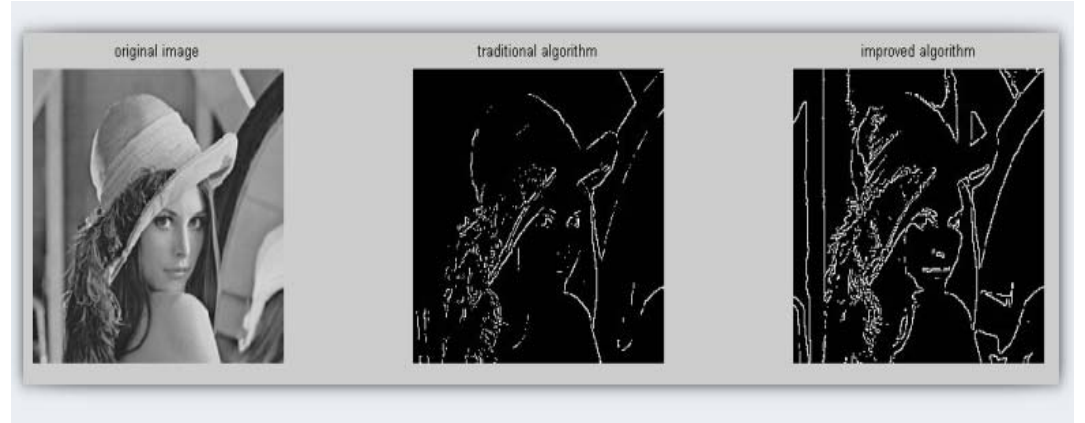

Figure 1. LENA edge detection.

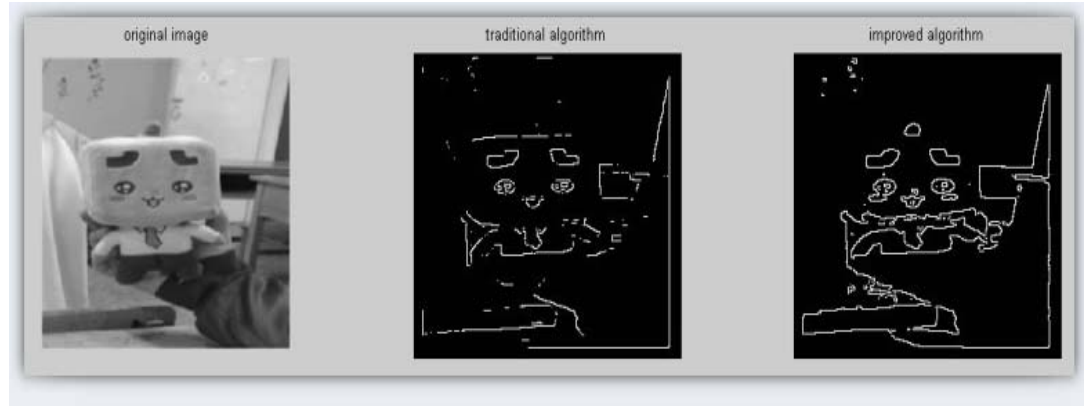

Figure 2. FANG edge detection.

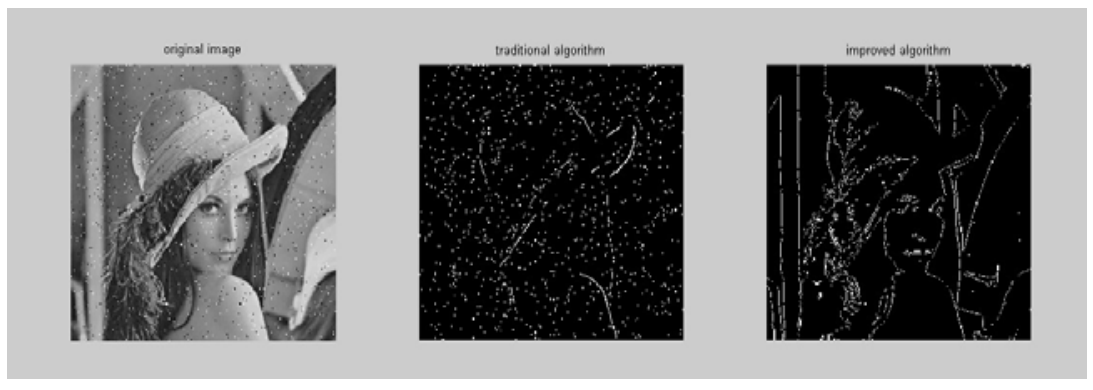

Figure 3. LENA after adding noise edge detection.

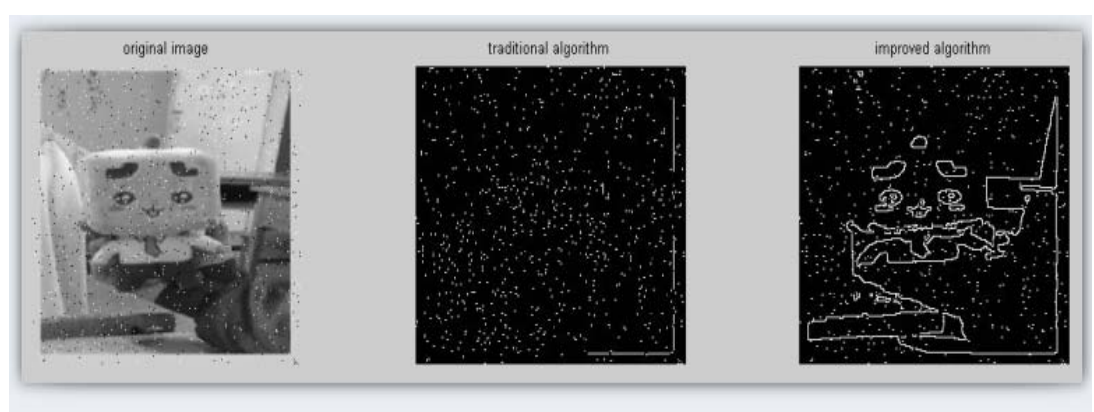

Figure 4. FANG after adding noise edge detection. 